\title{
A General Range-Velocity Processing Scheme for Discontinuous Spectrum FMCW Signal in HFSWR Applications
}

\author{
Mengguan Pan, ${ }^{1,2}$ Baixiao Chen, ${ }^{1,2}$ and Minglei Yang ${ }^{1,2}$ \\ ${ }^{1}$ National Laboratory of Radar Signal Processing, Xidian University, Xian 710071, China \\ ${ }^{2}$ Collaborative Innovation Center of Information Sensing and Understanding at Xidian University, Xi'an, China \\ Correspondence should be addressed to Baixiao Chen; bxchen@xidian.edu.cn
}

Received 5 May 2016; Accepted 15 August 2016

Academic Editor: Xiongbin Wu

Copyright (c) 2016 Mengguan Pan et al. This is an open access article distributed under the Creative Commons Attribution License, which permits unrestricted use, distribution, and reproduction in any medium, provided the original work is properly cited.

\begin{abstract}
Discontinuous spectrum signal which has separate subbands distributed over a wide spectrum band is a solution to synthesize a wideband waveform in a highly congested spectrum environment. In this paper, we present a general range-velocity processing scheme for the discontinuous spectrum-frequency modulated continuous wave (DS-FMCW) signal specifically. In range domain, we propose a simple time rearrangement operation which converts the range transform problem of the DS-FMCW signal to a general spectral estimation problem of nonuniformly sampled data. Conventional periodogram results in a dirty range spectrum with high sidelobes which cannot be suppressed by traditional spectral weighting. In this paper, we introduce the iterative adaptive approach (IAA) in the estimation of the range spectrum. IAA is shown to have the ability to provide a clean range spectrum. On the other hand, the discontinuity of the signal spectrum has little impact on the velocity processing. However, with the range resolution improved, the influence of the target motion becomes nonnegligible. We present a velocity compensation strategy which includes the intersweep compensation and in-sweep compensation. Our processing scheme with the velocity compensation is shown to provide an accurate and clean range-velocity image which benefits the following detection process.
\end{abstract}

\section{Introduction}

High frequency surface wave radar (HFSWR) refers to a classification of radar that operates in the HF band (3$30 \mathrm{MHz}$ ) and utilizes the surface wave mode of propagation. These systems can be used both in surveillance to detect and track vessels and in oceanography to measure surface current and ocean wave parameters [1-6].

However, the HF band is a heavily congested part of the radio spectrum which makes it difficult to find a continuous silent frequency band to transmit radar signals. This limits the signal bandwidth of the HF radar system and results in a poor range resolution [7, 8]. In [7], a continuous measurement of noise and interference data in the frequency band of 3-6 MHz at Cape Race, Newfoundland, Canada, in the period between August 1, 1998, and May 10, 2000, shows that channels with a bandwidth of $20 \mathrm{kHz}$ are readily available and a bandwidth of $100 \mathrm{kHz}$ is not available.
To overcome the trouble of limited band, Green and Kutuzov proposed a waveform design idea which utilizes several discontinuous but clean subbands to synthesize a signal with the desired bandwidth [9-11]. The discontinuity of the signal spectrum leads to high sidelobe levels of the matched filter output which cannot be suppressed by conventional spectral weighting. Therefore, most of the efforts at present focused on applying the sidelobe suppression technique in the matched filtering output. The first category of the methods is the mismatched filter (or instrumental variable filter) approach. It is a data-independent method which gets the filter coefficients by optimizing the autocorrelation function (ACF) and applies the filter to the matched filter output. The second category uses the CLEAN algorithm to reconstruct the target signal [12]. The third category is based on spectrum reconstruction; this includes using AR model $[9,13]$ or regularization interpolation [12] to fill the "gap" parts of the spectrum. And, on the other hand, [14-16] considered 
suppressing the sidelobes from a perspective of waveform designing rather than signal processing.

While some papers use the model of frequency hopped pulse signal [16-18], some other papers consider a more abstract discontinuous spectrum signal model and concentrate on the analysis of the autocorrelation function and the power spectrum density (PSD) $[14,15]$; only a few papers use a signal model of FMCW [12,13]. As the FMCW signal [19] and its variation FMICW signal [20] are widely used in HF radar applications such as the SeaSonde [3] and WERA [5] systems and they are quite different from the pulsed signal both in receiver structure and following signal processing method, we focus on the processing of discontinuous spectrum FMCW (DS-FMCW) signal specifically. Similar to the conventional FMCW signal, DS-FMCW uses stretch processing instead of correlation processing used in pulsed radar in the range domain. And we call this process range transform compared to the commonly used term "pulse compression" in pulsed radar.

Different from the conventional FMCW radar which outputs a constant-frequency complex sinusoid (beat signal) after mixing with the transmitted reference signal, the DSFMCW radar outputs a constant-frequency signal but with phase hopped at specific locations. In this paper, we propose a kind of transformation applied on the time variable, by which the hopped phase is converted to a kind of "hopped" sampling instants. This process may be implicit in former works $[12,13]$ and we choose to call it time rearrangement operation here. After the time rearrangement operation, the sampled postmixing signal becomes a nonuniformly sampled sequence of a complex sinusoid. Therefore, we set up a general spectral analysis model for the range processing of the DSFMCW signal and the crux of the matter now becomes the spectral estimation of a nonuniformly sampled data sequence.

The spectral analysis of nonuniformly sampled data has drawn much attention since the nonuniformity in the data is common in various applications [21]. And a large number of methods have emerged recently. The classical Fourier transform based periodogram suffers from the heavy leakage problem which leads to a low resolution and high sidelobe levels. Therefore, it is not applicable in the processing of DS-FMCW signal. In this paper, we apply the iterative adaptive approach (IAA) algorithm in the range spectrum estimation of the DS-FMCW signal. IAA was first proposed in [22] for target direction of arrival (DOA) estimation. It can be interpreted as an iteratively weighted least-square periodogram which eliminates almost completely the leakage problems of the conventional periodogram method in a fully data-adaptive manner [22, 23]. It is quite suitable to be applied in the range spectrum estimation of DS-FMCW signal. However, other spectral analysis methods suitable for the line spectrum estimation can also be taken into consideration. We use IAA as an example in this paper.

We consider the range-velocity processing of the DSFMCW signal afterwards. With the range resolution improved, some subtle issues may occur due to target motion. Accordingly, we propose a velocity compensation strategy in

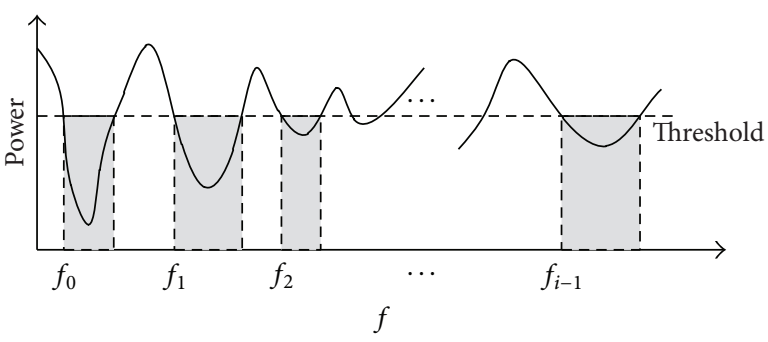

FIGURE 1: HF spectrum distribution illustration.

this paper. Finally, we present a general range-velocity processing scheme for the DS-FMCW signal.

This paper is organized as follows. Section 2 formulates the signal model and the range processing model of the DS-FMCW signal. The time rearrangement operation is introduced in this section. Section 3 extends the model to range-velocity processing and gives a velocity compensation strategy. A general range-velocity processing scheme for the DS-FMCW signal is presented at the end of this section. Section 4 gives a design example of a DS-FMCW signal and shows the effectiveness of our proposed processing scheme by several experiments. The final section concludes this paper.

\section{DS-FMCW Signal Model and Range Transform}

2.1. Signal Model and Time Rearrangement Operation. The HF radar system using discontinuous spectrum signal should cooperate with a spectrum monitor which monitors the spectrum environment before the radar starts to transmit a signal. A typical HF spectrum distribution is illustrated in Figure 1 . The shading areas below the threshold correspond to the silent frequency bands which can be used by the radar. There are $I$ relative silent segments in the frequency span $f_{0}$ to $f_{0}+\Delta F$. Let the start frequency and start time of each segment be $f_{i}$ and $t_{i}, i=0,1, \ldots, I-1, t_{0}=0$. Then, the duration of each segment is $T_{i}=t_{i+1}-t_{i}, i=0,1, \ldots, I-1$, and the sweep period is $T_{m}=\sum_{i=0}^{I-1} T_{i}$. We use a linear frequency sweep in each segment and fix the sweep slope to $\mu=$ $B / T_{m}$, in which $B$ is the total frequency bandwidth occupied by the radar signal. The ratio of the occupied frequency bandwidth of the radar signal to the whole frequency span is defined as the occupied frequency ratio (OFR) of the signal. Thus, OFR $=B / \Delta F$. The resulting operation waveform is the so-called discontinuous spectrum-frequency modulated continuous wave (DS-FMCW) signal. The transmitted and received signal frequency time characteristics are shown in Figure 2.

The transmitted signal in a single sweep period is

$$
\begin{aligned}
s(t)= & \sum_{i=0}^{I-1} \operatorname{rect}\left(\frac{t-T_{i} / 2-t_{i}}{T_{i}}\right) \exp \left(j \pi \mu\left(t-t_{i}\right)^{2}\right) \\
& \cdot \exp \left(j 2 \pi f_{i}\left(t-t_{i}\right)\right)
\end{aligned}
$$




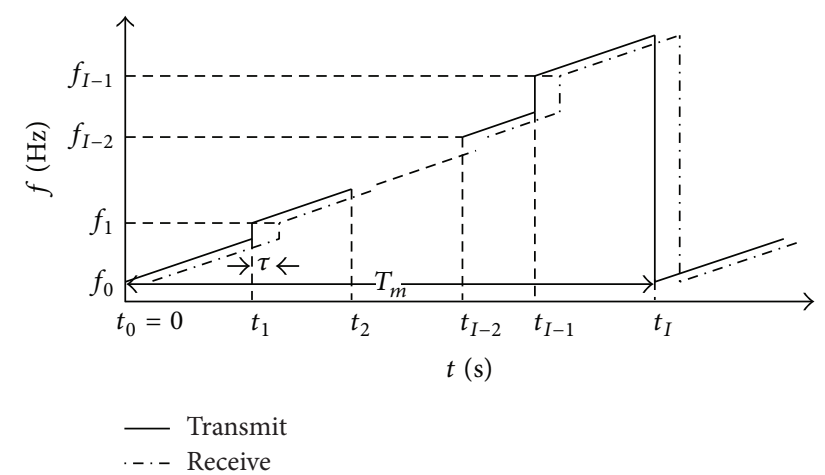

Figure 2: DS-FMCW transmitted/received signal frequency time characteristic.

where

$$
\operatorname{rect}\left(\frac{t}{T}\right)= \begin{cases}1, & t \leq\left|\frac{T}{2}\right| \\ 0, & \text { others }\end{cases}
$$

is a rectangular window function. The received signal from a single target is an attenuated, delayed, and Doppler-shifted version of the transmitted signal. In this section, we only consider a stationary point target and leave the discussion of the Doppler/velocity processing to the next section. Then, the received signal is given by

$$
\begin{aligned}
& y(t) \\
& =A \sum_{i=0}^{I-1} \operatorname{rect}\left(\frac{t-T_{i} / 2-t_{i}}{T_{i}}\right) \exp \left(j \pi \mu\left(t-t_{i}-\tau\right)^{2}\right) \\
& \quad \cdot \exp \left(j 2 \pi f_{i}\left(t-t_{i}-\tau\right)\right)
\end{aligned}
$$

in which $A$ represents the attenuation factor which is mainly related to the target radar cross section (RCS) and $\tau$ is the round-trip time for the signal to propagate from the radar system and back. A multitarget case can be seen as a superposition of the different echoes at the receiver. Note that the effect of the target delay on the rectangular envelope is omitted since in HF radar applications the maximum potential target delay usually satisfies $\tau_{\max } \ll T_{m}[19]$.

Similar to the stretch processing of the conventional FMCW signal, the received signal is mixed with the transmitted signal at the receiver. This process is also called deramp processing or dechirp processing. Stretch processing is a technique to reduce the sampling-rate requirements of the following analog-to-digital converter (ADC) [24]. The output of the mixer is

$$
\begin{aligned}
x(t) & =y(t) s^{*}(t) \\
& =A \sum_{i=0}^{I-1} \operatorname{rect}\left(\frac{t-T_{i} / 2-t_{i}}{T_{i}}\right) \exp \left(j \phi_{i}(t)\right)
\end{aligned}
$$

in which the phase term of the $i$ th segment $\phi_{i}(t)$ is

$$
\phi_{i}(t)=2 \pi\left[-\left(f_{i}-\mu t_{i}\right) \tau-\mu \tau t+0.5 \mu \tau^{2}\right],
$$

$$
0 \leq i \leq I-1 \text {. }
$$

Unlike the mixer output of the conventional FMCW signal, which is a constant-frequency complex sinusoid (beat signal), the corresponding output of the DS-FMCW signal is more analogous to a kind of phase-coded signal with $I$ subpulses of each having a different initial phase related to the target delay. Thus, the range spectrum cannot be obtained by a direct FFT/IFFT operation. However, with a simple transform of (5),

$$
\phi_{i}(t)=-2 \pi \mu \tau\left(t+\frac{f_{i}}{\mu}-t_{i}\right)+\pi \mu \tau^{2}, \quad 0 \leq i \leq I-1,
$$

the unknown phase hopping quantity converts to a time domain shift related to the waveform parameters which are predefined. Therefore, a rearrangement of the time variable in each segment eliminates the phase hopping. The time vector after the rearrangement operation is

$$
t^{\prime}=t+\frac{f_{i}}{\mu}-t_{i}, \quad t_{i} \leq t<t_{i+1} .
$$

For each segment, we have

$$
\begin{array}{ccc}
t \in\left[t_{0}, t_{1}\right) & \longrightarrow & t^{\prime} \in\left[t_{0}, t_{1}\right), \\
t \in\left[t_{1}, t_{2}\right) & \longrightarrow & t^{\prime} \in\left[\frac{f_{1}}{\mu}, t_{2}-t_{1}+\frac{f_{1}}{\mu}\right), \\
\vdots & \vdots & \vdots \\
t \in\left[t_{I-1}, t_{I}\right) & \longrightarrow & t^{\prime} \in\left[\frac{f_{I-1}}{\mu}, t_{I}-t_{I-1}+\frac{f_{I-1}}{\mu}\right) .
\end{array}
$$

When the receiver samples the deramped signal at a uniform interval $T_{s}$ and by letting $t_{n}, 0 \leq n<N, N=$ $\left\lfloor T_{m} / T_{s}\right\rfloor$ be the sampling instants $(\lfloor\rfloor$ represents the notion of being rounded down), the corresponding sampling instants after the time rearrangement $t_{n}^{\prime}$ turn out to be nonuniform (or, more accurately, piecewise uniform). This process of time rearrangement is illustrated in Figure 3. Figure 3(b) shows that the phase hopping is eliminated after the rearrangement.

Accordingly, the sampled deramped signal can be represented as

$$
\begin{aligned}
x[n] & =A \exp (j \psi) \exp \left(-j 2 \pi \mu \tau\left(t_{n}^{\prime}\right)\right) \\
& =\widetilde{s} \cdot \exp \left(-j 2 \pi \mu \tau\left(t_{n}^{\prime}\right)\right),
\end{aligned}
$$

$$
0 \leq n<N \text {, }
$$

in which $\psi=\pi \mu \tau^{2}$ is a constant phase term and $\widetilde{s}=$ $A \exp (j \psi)$ represents a complex amplitude.

From (9), the sampled time series $x[n]$ is a constantfrequency complex sinusoid signal sampled at time $t_{n}^{\prime}$. Thus, the range transform problem of the DS-FMCW signal converts to the problem of spectral estimation of nonuniformly sampled data.

We can denote the sampled signal in vector form as

$$
\mathbf{x}=[x[0], x[1], \ldots, x[N-1]]^{T}=\widetilde{s} \cdot \mathbf{a}(\tilde{f}),
$$

where $(\cdot)^{T}$ represents the transpose operation, $\mathbf{x} \in \mathbb{C}^{N \times 1}$, $\tilde{f}=\mu \tau \in F \subset \mathbb{R}$ is the beat frequency which is proportional 


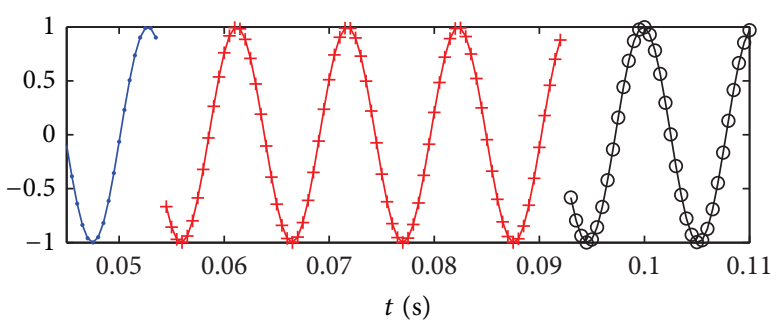

$\rightarrow$ Segment 1
+ Segment 2
$\rightarrow$ Segment 3

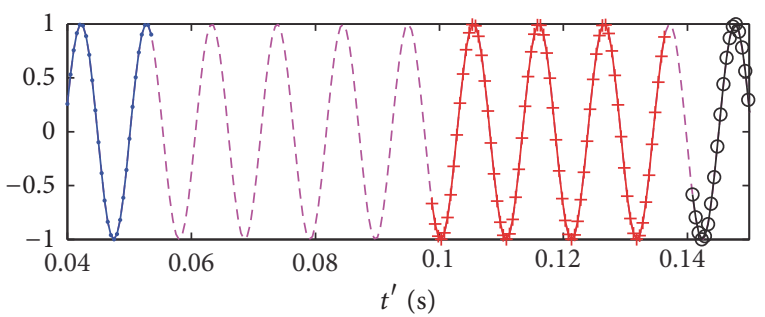

$\rightarrow$ Segment 1

1 Segment 2

- - Segment 3

(a) Prerearrangement

(b) Postrearrangement

FIGURE 3: Time rearrangement illustration.

to target delay $\tau$, in which $F$ is the considered frequency range, and $\mathbf{a}(\cdot): F \rightarrow \mathbb{C}^{N \times 1}$ is a known function which solely depends on the spectrum distribution of the DSFMCW signal; the function output is the matched filter vector (similar to the steering vector in the spatial processing) in the range domain

$$
\mathbf{a}(f)=\left[\begin{array}{c}
\exp \left(-j 2 \pi f t_{0}^{\prime}\right) \\
\exp \left(-j 2 \pi f t_{1}^{\prime}\right) \\
\vdots \\
\exp \left(-j 2 \pi f t_{N-1}^{\prime}\right)
\end{array}\right] .
$$

Consider the multitarget situation; the sampled data sequence of the deramped signal is

$$
\mathbf{x}=\sum_{l=1}^{L} \mathbf{a}\left(\widetilde{f}_{l}\right) \widetilde{s}_{l}+\boldsymbol{\epsilon}
$$

where $\boldsymbol{\epsilon} \in \mathbb{C}^{N \times 1}$ is a noise term and $L$ is the unknown number of targets. Equation (12) is a common model of spectral analysis of nonuniformly (irregularly) sampled data.

To assist in explaining the spectral analysis algorithms, we extend the model of (12). Let the considered frequency range be $F=\left\{f: f_{\min } \leq f \leq f_{\max }\right\}$, in which the maximum and the minimum beat frequencies are decided by the considered range window:

$$
\begin{aligned}
& f_{\max }=\mu \frac{2 R_{\max }}{c}, \\
& f_{\min }=\mu \frac{2 R_{\min }}{c} .
\end{aligned}
$$

We then use a uniform grid $\left\{f_{k}\right\}_{k=1}^{K}$ to cover $F$, with a specific fine step, and assume that $\left\{\tilde{f}_{l}\right\}$ lie on (practically, close to) the grid. This means that there exist $k_{1}, \ldots, k_{L}$ such that $\tilde{f}_{l}=$ $f_{k_{l}}, l=1, \ldots, L$. Also, let

$$
\begin{aligned}
& \mathbf{a}_{k}=\mathbf{a}\left(f_{k}\right), \quad k=1, \ldots, K, \\
& \boldsymbol{s}_{k}= \begin{cases}\widetilde{s}_{l}, & k=k_{l}(l=1, \ldots, L) \\
0, & \text { elsewhere. }\end{cases}
\end{aligned}
$$

Using this notation, we can rewrite (12) as

$$
\mathbf{x}=\sum_{k=1}^{K} \mathbf{a}_{k} s_{k}+\boldsymbol{\epsilon}
$$

2.2. Periodogram Range Spectrum. The most intuitive method is the classical Fourier transform based periodogram (FP), which is also known as single-frequency least-square (SFLS) method, matched filtering method, or beam-forming method (in array signal processing applications). It uses filter coefficients matched to the received signal structure, which yields the optimum output signal-to-noise ratio (SNR). The periodogram method can also be derived from a solution to the following least-squares (LS) data fitting problem:

$$
\widehat{s}(f)=\arg \min _{s(f)}\|\mathbf{x}-s(f) \mathbf{a}(f)\|^{2} .
$$

The solution to the above problem is

$$
\widehat{s}(f)=\frac{\mathbf{a}^{H}(f) \mathbf{x}}{\|\mathbf{a}(f)\|^{2}} .
$$

Then, the spectrum estimation at the frequency grid $\left\{f_{k}\right\}_{k=1}^{K}$ is given by

$$
\widehat{s}_{k}=\frac{\mathbf{a}_{k}^{H} \mathbf{x}}{\left\|\mathbf{a}_{k}\right\|^{2}}, \quad k=1, \ldots, K .
$$

The resolution of the periodogram method is proportional to the reciprocal of the observation time interval. 
According to (7), in the DS-FMCW situation, the observation interval after a rearrangement of the sampling instants is

$$
\begin{aligned}
\Delta t & =t_{N-1}^{\prime}-t_{0}^{\prime}=T_{m}+\frac{f_{I-1}}{\mu}-t_{I-1} \\
& =\frac{\left(f_{I-1}+\mu\left(T_{m}-t_{I-1}\right)\right)}{\mu}=\frac{\Delta F}{\mu},
\end{aligned}
$$

where $\Delta F$ is the total frequency span. Thus, the observation interval is stretched from $T_{m}=B / \mu$ to $\Delta F / \mu$ after the rearrangement of the sampling instants. Then, the range resolution is given by

$$
\Delta R=\frac{1}{\Delta t \cdot \mu} \cdot \frac{c}{2}=\frac{c}{2 \Delta F} .
$$

Therefore, the range resolution solely depends on the total frequency span $(\Delta F)$ of the DS-FMCW signal. By adopting the waveform in an appropriate frequency span, we can obtain the desired range resolution. However, the development of the spectral analysis has led to many methods which can resolve targets whose range separation is within $\Delta R$. Therefore, we call the resolution of periodogram the inherent resolution.

However, the spectrum discontinuity in the frequency span leads to high sidelobes when applying the conventional periodogram method which cannot be suppressed by conventional spectral weighting. The sidelobe levels are related to the waveform occupied frequency ratio (OFR) and the specific spectrum distribution in the frequency span. The larger the OFR and the more uniform the distribution, the lower the sidelobe levels. In this paper, we do not consider extreme cases such as the occupied frequency being at the two sides of the frequency span or being all concentrated into a large continuous block, in which the situation degrades to a continuous spectrum. We assume the occupied frequency is distributed uniformly in the frequency span which is more likely encountered in the practical situation.

2.3. IAA Range Spectrum. The inherent limitations of the periodogram approach motivate a more effective spectral analysis method to be applied here. IAA is a nonparametric and user parameter free weighted least-squares based spectral analysis method first proposed for spatial spectral estimation [22]. It is a periodogram related spectral analysis method which eliminates almost completely the leakage problems of the periodogram method in a fully data-adaptive manner. The range spectrum estimation problem is equivalent to a direction of arrival (DOA) estimation problem with single snapshot in the array processing applications. And IAA can properly handle this situation. Consequently, IAA is quite suitable to be applied in the situation of range spectrum estimation of the DS-FMCW signal.

Periodogram uses solution to the LS fitting problem (16) as the estimate in each frequency scan point. It is a dataindependent approach. While estimating a spectrum value of a specific frequency point $f_{k}$, the other components located at frequencies different from $f_{k}$ perform as interferences.
Prewhitening of the other frequency components except the current processing one can cancel strong interferences in the data. Therefore, IAA uses a weighted least-squares (WLS) fitting criterion with the weight matrix equal to the inversion of the interferences and noise covariance matrix:

$$
\widehat{s}_{k}=\arg \min _{s_{k}}\left\|\mathbf{x}-s_{k} \mathbf{a}_{k}\right\|_{\mathbf{R}_{i+n, k}^{-1}}^{2}, \quad k=1, \ldots, K
$$

in which $\mathbf{R}_{i+n, k}$ is the interferences (signals at range cells other than the range cell $k$ of current interest) and noise covariance matrix and square of the weighted $\ell_{2}$-norm is defined as $\|\mathbf{x}\|_{\mathbf{W}}^{2} \triangleq \mathbf{x}^{H} \mathbf{W} \mathbf{x}$.

The iterative solution to the WLS problem (21) leads to the IAA algorithm. As the range transform problem of the DS-FMCW signal is equivalent to a general spectral analysis problem of nonuniformly sampled data, the standard IAA algorithm can be directly applied here [22, 23].

\section{Extension to Range-Velocity Processing}

Conventional FMCW radar uses Fast Fourier Transform (FFT) on the range domain (fast time) and pulse domain (slow time), respectively, to form a range-Doppler spectrum (RD image), thus attaining the target range and Doppler information. In the last section, we have proposed that the range transform of the DS-FMCW signal is equivalent to a spectral estimation problem of a nonuniformly sampled data set. Then, we can replace the FFT adopted by FMCW in the range domain processing with a more effective spectral analysis method. However, the spectrum discontinuity of the waveform has little impact on the Doppler processing. We can still employ FFT in the Doppler processing which is essentially a matched filter bank. But since the carrier frequency of the signal changes at different time segments, a single target's velocity corresponds to different Doppler frequencies. Therefore, we use a matched filter bank in the velocity domain directly which is more intuitive than processing in the Doppler domain and then adjusting different Doppler cells into a single velocity cell according to their carrier frequencies. This is the reason why we use the term range-velocity processing instead of the commonly used term range-Doppler processing.

In this section, we present a scheme for the range-velocity processing of the DS-FMCW signal. We change the sequence of processing steps with applying velocity domain matched filtering first followed by range domain spectral analysis at each velocity channel. This modification has the following benefits:

(1) The matched filter coefficients in the velocity domain for each range bin can be easily decided since their carrier frequency is predefined.

(2) After velocity processing, we can compensate the range bias caused by the Doppler shift in a single sweep period by multiplying a correction factor in each velocity channel. This bias is usually omitted in the RD processing of conventional FMCW HF radar. 
(3) If we choose a spectral analysis method which does not reserve the phase information at the output, we have to do the velocity processing first.

Suppose there are $M$ sweep periods in a coherent processing interval (CPI); the transmitted signal can be expressed as

$$
\begin{aligned}
s(t)= & \sum_{m=0}^{M-1} \sum_{i=0}^{I-1} \operatorname{rect}\left(\frac{t-T_{i} / 2-t_{i}-m T_{m}}{T_{i}}\right) \\
& \cdot \exp \left(j \pi \mu\left(t-t_{i}-m T_{m}\right)^{2}\right) \\
& \cdot \exp \left(j 2 \pi f_{i}\left(t-t_{i}-m T_{m}\right)\right) .
\end{aligned}
$$

The received signal from a moving target can be expressed as

$$
\begin{aligned}
y(t)= & A \sum_{m=0}^{M-1} \sum_{i=0}^{I-1} \operatorname{rect}\left(\frac{t-T_{i} / 2-t_{i}-m T_{m}}{T_{i}}\right) \\
& \cdot \exp \left(j \pi \mu\left(t-t_{i}-m T_{m}-\tau(t)\right)^{2}\right) \\
& \cdot \exp \left(j 2 \pi f_{i}\left(t-t_{i}-m T_{m}-\tau(t)\right)\right) .
\end{aligned}
$$

The impact of the target delay on the signal envelope is omitted as before; $A$ represents the attenuation factor. And the delay function is

$$
\tau(t)=\frac{2\left(R_{0}-v t\right)}{c}=\tau_{0}-\frac{2 v t}{c},
$$

where $R_{0}$ is the initial range of the target, $\tau_{0}$ is the corresponding delay, and $v$ is the radial velocity of the target towards the radar. After mixing the received signal with the transmitted signal, we get the deramped output

$$
\begin{aligned}
x(t)= & y(t) s^{*}(t) \\
= & A \sum_{m=0}^{M-1} \sum_{i=0}^{I-1} \operatorname{rect}\left(\frac{t-T_{i} / 2-t_{i}-m T_{m}}{T_{i}}\right) \\
& \cdot \exp \left(-j 2 \pi f_{i} \tau(t)\right) \\
& \cdot \exp \left(-j 2 \pi \mu \tau(t)\left(t-t_{i}-m T_{m}\right)+j \pi \mu \tau^{2}(t)\right) \\
= & A \sum_{m=0}^{M-1} \sum_{i=0}^{I-1} \operatorname{rect}\left(\frac{t-T_{i} / 2-t_{i}-m T_{m}}{T_{i}}\right) \\
& \cdot \exp \left(j \phi_{m, i}(t)\right),
\end{aligned}
$$

in which the phase term is

$$
\phi_{m, i}(t)=-2 \pi \mu \tau(t)\left(t-t_{i}+\frac{f_{i}}{\mu}-m T_{m}\right)+\pi \mu \tau^{2}(t) .
$$

Let $\Delta t_{i}=f_{i} / \mu-t_{i}$ and substitute (24) into (26); we get

$$
\begin{aligned}
\phi_{m, i}(t)= & -2 \pi \mu \tau_{0}\left(t+\Delta t_{i}-m T_{m}\right) \\
& +2 \pi \mu \frac{2 v t}{c}\left(t+\Delta t_{i}-m T_{m}\right) \\
& +\pi \mu\left(\tau_{0}-\frac{2 v t}{c}\right)^{2} \\
\triangleq & \phi_{1(m, i)}(t)+\phi_{2(m, i)}(t)+\phi_{3(m, i)}(t) .
\end{aligned}
$$

Let $t_{1}$ be the time vector in a single sweep period; then the time vector in the $m$ th sweep period is $t=t_{1}+m T_{m}$. Thus, the first phase term is

$$
\phi_{1(m, i)}(t)=-2 \pi \mu \tau_{0}\left(t_{1}+\Delta t_{i}\right)
$$

which is the same as the stationary model (6) in Section 2. After a time rearrangement, we can get the range spectrum from this term using a spectral analysis method.

We next consider the third term of $\phi_{m, i}(t)$

$$
\begin{aligned}
\phi_{3(m, i)}(t) & =\pi \mu \tau_{0}^{2}-2 \pi \mu \tau_{0} \frac{2 v t}{c}+\pi \mu \frac{4 v^{2} t^{2}}{c^{2}} \\
& \triangleq \phi_{3(m, i)}^{(1)}(t)+\phi_{3(m, i)}^{(2)}(t)+\phi_{3(m, i)}^{(3)}(t) .
\end{aligned}
$$

We have three contributions to the phase: a constant, a linear term in time $t$, and a quadratic term in time, $t^{2}$. Firstly, consider the phase variation of the quadratic term in a CPI. Assume that the target's distance of movement in a CPI is less than $3 \mathrm{~km}$; then, $v t / c<10^{-5}$. And the sweep slope of a HF FMCW signal is usually at the order of $\left(10^{6}\right)$; thus, the variation of the quadratic term $\phi_{3(m, i)}^{(3)}(t)$ in a CPI is at the order of $2 \pi \times 10^{-4}$, which can be omitted. Then, consider the linear term $\phi_{3(m, i)}^{(2)}(t)=-2 \pi \mu \tau_{0}(2 v t / c)$, which is proportional to the target velocity. As we will see in the following analysis, $\phi_{2(m, i)}$ (see (33)) is the so-called Doppler term and has the same form as $\phi_{3(m, i)}^{(2)}$. Since $\mu \tau_{0} \ll f_{0}$, the phase term $\phi_{3(m, i)}^{(3)}$ can also be omitted. In conclusion,

$$
\phi_{3(m, i)}=\Psi_{3},
$$

where $\Psi_{3}$ is a constant.

For the second term of $\phi_{(m, i)}(t)$,

$$
\begin{aligned}
\phi_{2(m, i)}(t) & =2 \pi \mu \frac{2 v t}{c}\left(t-m T_{m}\right)+2 \pi \mu \frac{2 v t}{c} \Delta t_{i} \\
& \triangleq \phi_{2(m, i)}^{(1)}(t)+\phi_{2(m, i)}^{(2)}(t)
\end{aligned}
$$

in which the first phase term is

$$
\begin{aligned}
\phi_{2(m, i)}^{(1)}(t)= & 2 \pi \mu \frac{2 v\left(t-m T_{m}\right)^{2}}{c} \\
& +2 \pi \mu \frac{2 v m T_{m}}{c}\left(t-m T_{m}\right) \\
\triangleq & \phi_{2(m, i)}^{(1,1)}+\phi_{2(m, i)}^{(1,2)} .
\end{aligned}
$$


$\phi_{2(m, i)}^{(1,1)}$ represents the quadratic disturbance term caused by the target velocity during a sweep period. Its phase variation during a sweep period is at the order of $\left(2 \pi \times 10^{-4}\right) \cdot\left(\phi_{2(m, i)}^{(1,2)}\right)$ has the same form as the intersweep Doppler term (the term $\left(\phi_{2(m, i)}^{(2,2)}(t)\right)$ in $\left.(33)\right)$, and $\left(\mu\left(t-m T_{m}\right)\right)$ is comparable to signal carrier frequency; thus, we should consider it in matched filtering in the velocity domain.

Lastly, the second term of $\left(\phi_{2(m, i)}(t)\right)$

$$
\begin{aligned}
\phi_{2(m, i)}^{(2)}(t)= & 2 \pi \frac{2\left(f_{i}-\mu t_{i}\right) v t}{c} \\
= & 2 \pi \frac{2\left(f_{i}-\mu t_{i}\right) v}{c}\left(t-m T_{m}\right) \\
& +2 \pi \frac{2\left(f_{i}-\mu t_{i}\right) v}{c} m T_{m} \\
\triangleq & \phi_{2(m, i)}^{(2,1)}(t)+\phi_{2(m, i)}^{(2,2)}(t)
\end{aligned}
$$

is the so-called Doppler term. The term $\left(\phi_{2(m, i)}^{(2,1)}(t)\right)$ in $(33)$ is the in-sweep Doppler term. It will cause a range bias in range processing. And the term $\left(\phi_{2(m, i)}^{(2,2)}(t)\right)$ in $(33)$ is the intersweep Doppler term.
In conclusion, the terms influenced by target velocity are

$$
\begin{aligned}
\phi_{v(m, i)}= & 2 \pi \frac{2\left(f_{i}-\mu t_{i}\right) v}{c}\left(t-m T_{m}\right) \\
& +2 \pi \frac{2\left(f_{i}-\mu t_{i}\right) v}{c} m T_{m} \\
& +2 \pi \frac{2 \mu\left(t-m T_{m}\right) v}{c} m T_{m} .
\end{aligned}
$$

Note that the first term and the last term in (34) also exist in conventional FMCW radar, but, in HF applications, they are usually omitted as their impact is minor. However, the neglect of the third term will lead to both a velocity bias and a range bias and the neglect of the first term will lead to a range bias. Thus, we choose to compensate both terms in our system. As the compensation of the third term is handled along the slow time domain and the compensation of the first term is handled along the fast time domain, we call them intersweep compensation and in-sweep compensation, respectively.

Intersweep compensation can be achieved by merging the compensation coefficients into the velocity domain matched filter coefficients. Let the $n$th sampling point in the fast time domain be $t_{1}[n]=t\left[n+m T_{m}\right]-m T_{m}$; then, we can get the velocity domain matched filter coefficients at the point $t_{1}[n]$ :

$$
\mathbf{a}_{n}(v)=\left[\begin{array}{c}
0 \\
\exp \left(j 2 \pi \frac{2\left(f_{i}-\mu t_{i}\right) T_{m} v}{c}\right) \cdot \exp \left(j 2 \pi \frac{2 \mu t_{1}[n] T_{m} v}{c}\right) \\
\vdots \\
\exp \left(j 2 \pi \frac{2\left(f_{i}-\mu t_{i}\right)(M-1) T_{m} v}{c}\right) \cdot \exp \left(j 2 \pi \frac{2 \mu t_{1}[n](M-1) T_{m} v}{c}\right)
\end{array}\right] .
$$

$f_{i}$ and $t_{i}$ in $\mathbf{a}_{n}(v)$ have to be chosen according to current fast time point.

Let the considered velocity range be $V=\left\{v: v_{\min } \leq\right.$ $\left.v \leq v_{\max }\right\}$, and, similar to the range domain, we use a uniform grid $\left\{v_{p}\right\}_{p=1}^{P}$ to cover $V$. Since the $p$ th velocity channel after the velocity domain matched filtering represents the fast time echoes from all the targets with the specific velocity $v_{p}$, we can compensate the range bias caused by the target velocity according to the first term of $\phi_{v(m, i)}$ at each velocity channel. The in-sweep compensation coefficient $\mathbf{B}$ in the $n$th fast time sampling point of the $p$ th velocity channel is

$$
\begin{aligned}
B_{p, n}=\exp \left(2 \pi \frac{2\left(f_{i}-\mu t_{i}\right) v_{p}}{c} t_{1}[n]\right) & \\
& 1 \leq p \leq P, 1 \leq n \leq N,
\end{aligned}
$$

in which $f_{i}$ and $t_{i}$ are decided from the current fast time sampling point $t_{1}[n]$.

The block diagram of the DS-FMCW radar system is shown in Figure 4. We employ a stretch processing based receiver and signal processor. The signal processor part follows our proposed range-velocity processing scheme with the details shown in Algorithm 1. Consider a series of $M$ sweeps; after the postmixing sampling, we have the sampled sequence $y[n], n=0,1, \ldots, M N-1$. With each sweep placed as a row, we get $M \times N$ data matrix $\mathbf{Y}$. The samples in each row of $\mathbf{Y}$ are successive samples of the returns in a single sweep, that is, successive range bins. Each column represents a series of measurements from the same range bin over successive sweep periods. We denote the $m$ th row of a matrix $\mathbf{Y}$ by $\mathbf{Y}_{m \text {, }}$ and the $n$th column of a matrix $\mathbf{Y}$ by $\mathbf{Y}_{:, n}$. We also denote the velocity matched filtering matrix at the range bin $n$ by $\mathbf{A}_{n}$, which includes all the vectors at different velocity grids

$$
\mathbf{A}_{n} \triangleq\left[\mathbf{a}_{n}\left(v_{1}\right), \ldots, \mathbf{a}_{n}\left(v_{p}\right)\right]
$$

In Figure 4 and Algorithm $1, \mathbf{Z} \in \mathbb{C}^{P \times N}$ is the velocity domain matched filtering output. After the velocity compensation in each velocity channel, we get the compensated output $\mathbf{Z}^{(c)}=\mathbf{Z} \odot \mathbf{B}^{*} \in \mathbb{C}^{P \times N}$, where $\odot$ stands for the 


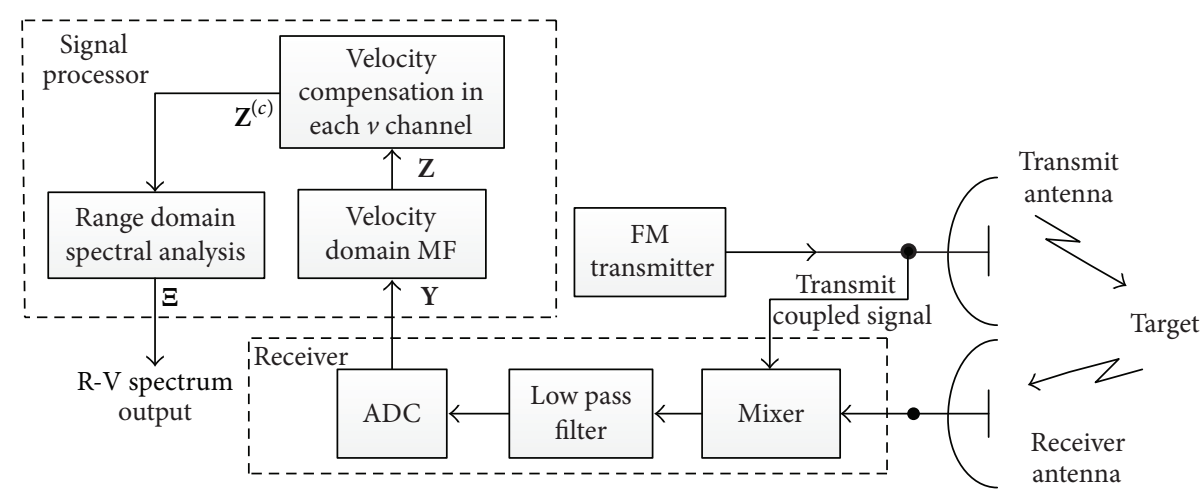

FIGURE 4: Block diagram of DS-FMCW radar system.

input: data matrix of $M$ sweeping periods $\mathbf{Y}$

step 1 velocity domain matched filtering

for each range bin $n$

$$
\mathbf{Z}_{:, n}=\mathbf{A}_{n}^{H} \mathbf{Y}_{:, n}
$$

endfor

step 2 velocity compensation in each velocity channel

$\mathbf{Z}^{(c)}=\mathbf{Z} \odot \mathbf{B}^{*}$

step 3 range spectrum estimation in each velocity channel for each velocity channel $p$

$\Xi_{p::}=$ applying spectral analysis to $\mathbf{Z}_{p,:}^{(c)}$

endfor

output: range-velocity spectrum $\Xi$

Algorithm 1: DS-FMCW range-velocity processing scheme.

Hadamard (element-wise) product and $(\cdot)^{*}$ denotes the conjugate operator. Finally, the spectral analysis in each velocity channel gives the final range-velocity spectrum $\Xi \in \mathbb{C}^{P \times K}$, in which $K$ is the range bin number that coincides with the range domain model in Section 2.

\section{Numerical Experiments}

In the first experiment, we demonstrate the determination of the postmixing sampling rate by using the spectral window method [25]. In the second experiment, we illustrate the range spectrum formed by the periodogram and the IAA algorithm. In the third experiment, we evaluate the performance of IAA when applying it in range domain spectral analysis as the OFR of the DS-FMCW signal varies by several Monte Carlo simulations. The second and third experiments only consider the range domain processing while the fourth experiment gives a complete range-velocity processing result in accordance with the processing scheme shown in Figure 4 and Algorithm 1.

In Experiments 1, 2, and 4, we use the same DS-FMCW signal designed according to Canada 1999 (summer) HF band spectrum monitoring result [7]; the available frequency band distribution is shown in Table 1.

The designed DS-FMCW signal utilizes all the clear bands between the start frequency $f_{0}=5.11 \mathrm{MHz}$ and the end
TABLE 1: Canada 1999 summer available spectrum.

\begin{tabular}{lcc}
\hline Channel & Frequency range $(\mathrm{MHz})$ & Bandwidth $(\mathrm{kHz})$ \\
\hline 1 & $5.11 \sim 5.14$ & 30 \\
2 & $5.26 \sim 5.27$ & 10 \\
3 & $5.29 \sim 5.31$ & 20 \\
4 & $5.37 \sim 5.39$ & 20 \\
5 & $5.46 \sim 5.48$ & 20 \\
6 & $5.51 \sim 5.52$ & 10 \\
7 & $5.54 \sim 5.59$ & 50 \\
8 & $5.64 \sim 5.66$ & 20 \\
9 & $5.72 \sim 5.73$ & 10 \\
10 & $5.77 \sim 5.80$ & 30 \\
\hline
\end{tabular}

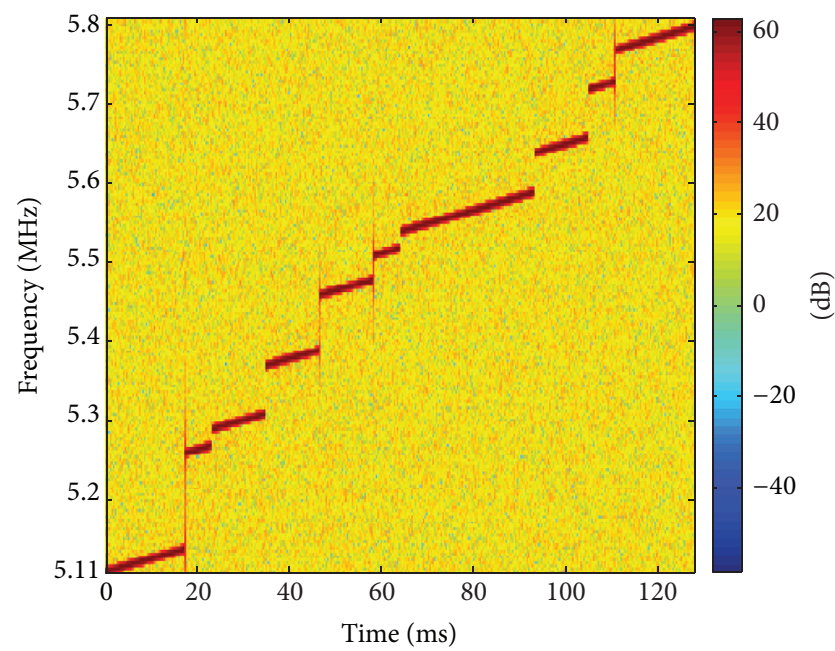

FIGURE 5: Time-frequency characteristic of the transmit signal.

frequency $f_{0}+\Delta F=5.80 \mathrm{MHz}$ as shown in Table 1 . Hence, it has the frequency span $\Delta F=690 \mathrm{kHz}$ and total clear bandwidth $B=220 \mathrm{kHz}$. Then, the inherent range resolution of the DS-FMCW signal is $\Delta R=c / 2 \Delta F=217 \mathrm{~m}$. And the sweep period $T_{m}$ is set to $128 \mathrm{~ms}$. The time-frequency characteristic of the transmitted signal is shown in Figure 5. The time is continuous and the frequency is hopped at specific locations. 


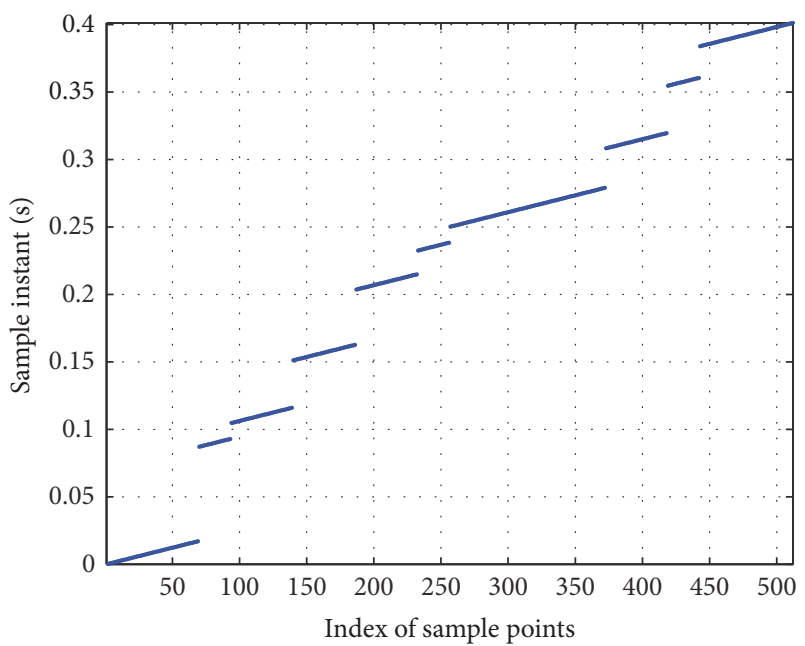

FIGURE 6: Sampling instants in a single sweep period after the time rearrangement operation.

\subsection{Experiment 1: Determination of Postmixing Sampling} Rate. In a stretch processing receiver, the sampling process comes after the mixer, by which the sampling rate can be much less than the signal bandwidth. In a conventional FMCW radar, the minimum postmixing sampling rate without any aliasing problem is proportional to the considered range window extent:

$$
F_{s}>\Delta F \cdot \frac{2 R_{\text {win }} / c}{T_{m}},
$$

where $R_{\text {win }}=R_{\max }-R_{\min }$. This is defined through the Nyquist-Shannon sampling theorem essentially. However, for the DS-FMCW signal, the sampling instants after the time rearrangement operation become nonuniform (Figure 6), and there is no well accepted definition of the nonaliasing sampling rate for the nonuniformly sampled data sequence. In this paper, we adopt the Nyquist or rollover frequency definition for nonuniform samples described in [25], which is called the spectral window method. Given $N$ nonuniform samples with sampling instants $\left\{t_{n}\right\}_{n=1}^{N}$, the spectral window at any frequency $f$ is defined as

$$
W(f)=\left|\frac{1}{N} \sum_{n=1}^{N} \exp \left(j 2 \pi f t_{n}\right)\right|^{2} .
$$

We can easily verify that $W(0)=1$, and the frequency range without any aliasing problem can be determined by examining the largest range for $f$ in which the only peak with height equal (or close) to 1 is at $f=0$.

For our designed DS-FMCW signal, at a postmixing sampling rate of $F_{s}=4 \mathrm{kHz}$, the sampling instants after the time rearrangement operation $\left(t_{n}^{\prime}\right)$ are shown in Figure 6. Figure 6 shows that the postrearrangement sampling instants $t_{n}^{\prime}$ are nonuniformly (more precisely, piecewise uniformly) distributed and total observation interval is stretched from the sweep period $T_{m}=128 \mathrm{~ms}$ to an equivalent virtual interval of $\Delta F / \mu=402 \mathrm{~ms}$. And, on the other hand,

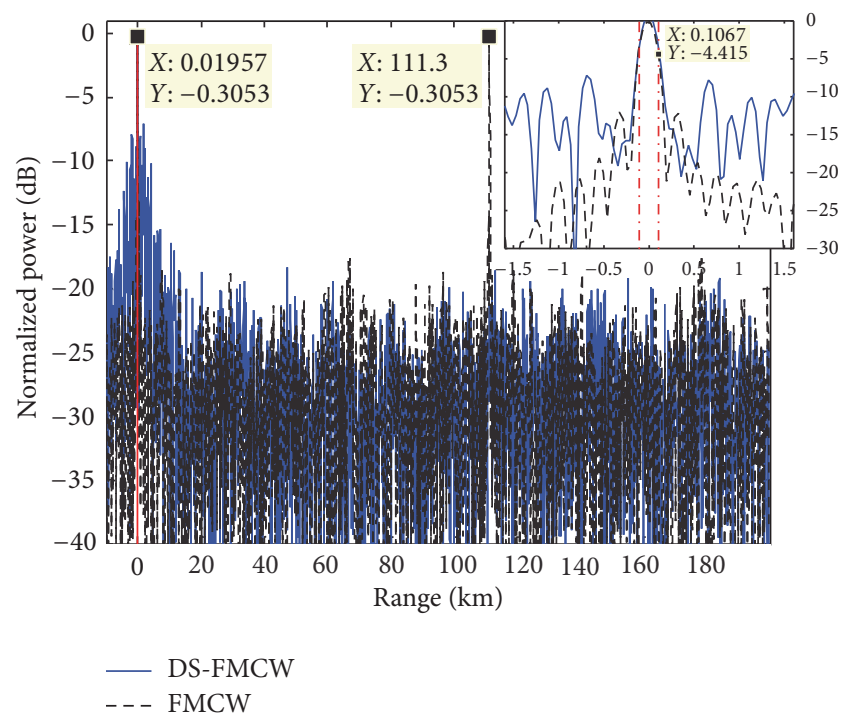

FIGURE 7: Spectral window of the designed DS-FMCW signal and a continuous FMCW signal within the same frequency span.

comparing with Figure 5, we can see that two figures have an identical distribution. This illustrates the role of the time rearrangement which is converting the discontinuity of the signal spectrum to the discontinuity of the sampling instants.

In fact, we can derive that the periodogram spectrum is just a superposition of spectral windows with different shift amount related to the target range. Thus, the spectral window and the periodogram spectrum have the same spectral shape. We use a range scan step $\delta R$ that is five times smaller than the traditional range resolution according to (20):

$$
\delta R=\frac{1}{5} \cdot \frac{c}{2 \Delta F} .
$$

The spectral window of the designed DS-FMCW signal is shown in Figure 7, and the spectral window of a full occupied FMCW signal in the same frequency span $\left(f_{0} \sim f_{0}+\Delta F\right)$ is plotted in the same figure for comparison.

In Figure 7, the spectral window of the FMCW signal is aliased in the range window $0 \sim 200 \mathrm{~km}$ while the DSFMCW signal is not. And the maximum range window for the FMCW signal is $111.3 \mathrm{~km}$ which is consistent with (38). Therefore, the spectral window can be used to ensure that the range spectrum of the DS-FMCW signal is unambiguous under the selected sampling rate.

Figure 7 also indicates that, compared with the conventional FMCW signal within the same frequency span, the range spectrum of the DS-FMCW signal has the same main lobe width $(c /(2 \Delta F))$ but has much higher sidelobe levels. Thus, other methods rather than periodogram should be considered to be applied in the range spectrum estimation of the DS-FMCW signal.

4.2. Experiment 2: Periodogram Range Spectrum and IAA Range Spectrum. In this experiment, we illustrate the improvement of the sidelobe levels and range resolution provided by IAA when applied in the range spectrum analysis. 


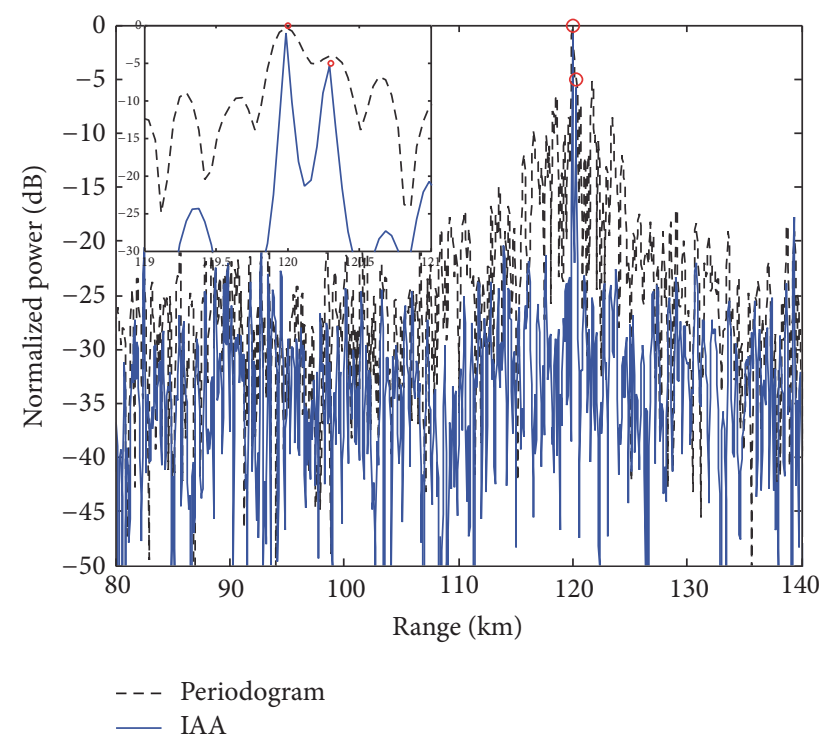

FIGURE 8: Range spectrum generated by periodogram and IAA.

We consider two targets located at the range of $120 \mathrm{~km}$ and $120.3 \mathrm{~km}$ whose separation is slightly larger than the inherent range resolution and with the input SNR of $0 \mathrm{~dB}$ and $-5 \mathrm{~dB}$, respectively. The range profiles generated by periodogram and IAA are shown in Figure 8. It can be inferred from Figure 8 that the periodogram method suffers from heavy leakage problems which make the peak of weaker target almost masked by the high sidelobes of a strong target nearby. And the high sidelobe level also causes false alarms and contaminates the detection output. IAA, on the other hand, resolves two closely separated targets clearly and has a much lower sidelobe level. The sidelobe level of the IAA spectrum can hardly be determined from Figure 8 as it is even lower than the noise power. In fact, the spectrum output of IAA is quite close to a line spectrum. There are sharp peaks at the locations of the targets and near-zero values at the other locations.

4.3. Experiment 3: Performance of the IAA Range Spectrum. This subsection designs two Monte Carlo experiments to investigate the performance of IAA when applied in the range processing of the DS-FMCW signal. In these two experiments, we use a similar waveform parameter of the DS-FMCW signal. We fix the total frequency span $\Delta F$ to $600 \mathrm{kHz}$. As stated at the end of Section 2, we do not consider the extreme situations and when we consider a specific OFR, we generate randomly a uniformly distributed discontinuous spectrum with total free bandwidth $B=\Delta F$. OFR at each Monte Carlo trial.

Firstly, we examine the peak sidelobe level (PSL) of the IAA range spectrum of the DS-FMCW signal as the OFR varies. PSL is defined as the ratio of the maximum sidelobe level to the main lobe level. In this experiment, we fix the SNR to $10 \mathrm{~dB}$. Figure 9 shows the PSL of periodogram and IAA range spectrum via 500 Monte Carlo trials when the OFR varies from $20 \%$ to $100 \%$. We can infer from the figure that

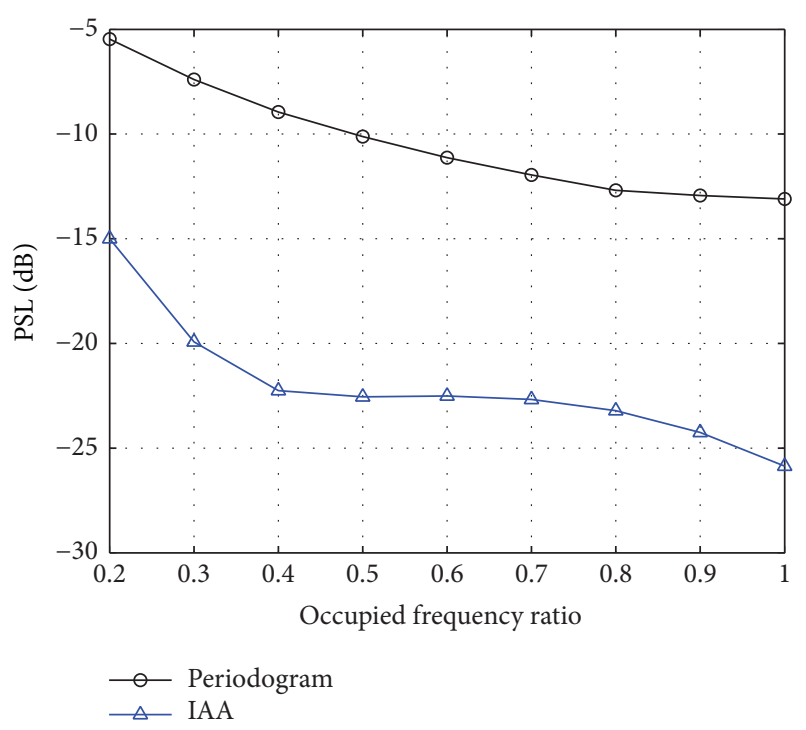

FIGURE 9: PSL of IAA range spectrum versus OFR.

TABLE 2: Simulated target information.

\begin{tabular}{lccc}
\hline Index & Range $(\mathrm{km})$ & Velocity $(\mathrm{km} / \mathrm{h})$ & SNR $(\mathrm{dB})$ \\
\hline 1 & 120 & 40 & 0 \\
2 & 120 & 50 & -5 \\
3 & 120.3 & 40 & 0 \\
4 & 120.3 & 50 & 0 \\
5 & 120 & 0 & 0 \\
\hline
\end{tabular}

as the PSL of IAA spectrum can be lower than $-20 \mathrm{~dB}$ with an OFR larger than $40 \%$, the PSL of periodogram is larger than $-13 \mathrm{~dB}$ at any OFR and cannot be suppressed via spectral weighting when the OFR is less than 1.

The second Monte Carlo simulation examines the capability of resolving closely spaced targets by IAA. Two targets are placed at ranges $120 \mathrm{~km}$ and $120.2 \mathrm{~km}$, whose separation is slightly less than the inherent range resolution. By definition, two targets are resolved in a given run if both $\left|\widehat{R}_{1}-R_{1}\right|$ and $\left|\widehat{R}_{2}-R_{2}\right|$ are smaller than $\left|R_{1}-R_{2}\right| / 2$, where $\widehat{R}_{k}$ and $R_{k}$ denote the estimated and true range for the $k$ th target, respectively. Figure 10 shows that, at a SNR higher than $0 \mathrm{~dB}$ and an OFR higher than $40 \%$, IAA can properly resolve the targets.

\subsection{Experiment 4: A Complete Range-Velocity Processing} Example of DS-FMCW Signal. In this subsection, we demonstrate the whole range-velocity process shown in Figure 4 and Algorithm 1. The velocity domain matched filtering is done first followed by correcting in each velocity channel the phase offset caused by the target velocity in a single sweep period (in-sweep compensation), and the range spectrum analysis is then done in each velocity channel to output the final rangevelocity spectrum.

We consider five targets whose information is shown in Table 2. The first to the fourth targets are closely spaced both in range and in velocity domain, and the fifth target 


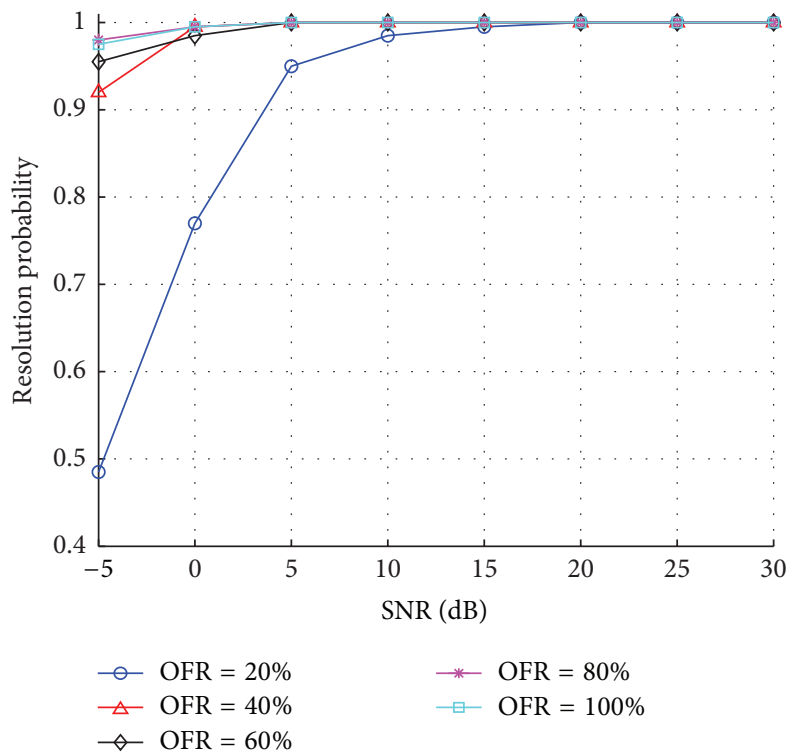

FIGURE 10: IAA resolution probability versus SNR for closely located targets at different OFR.

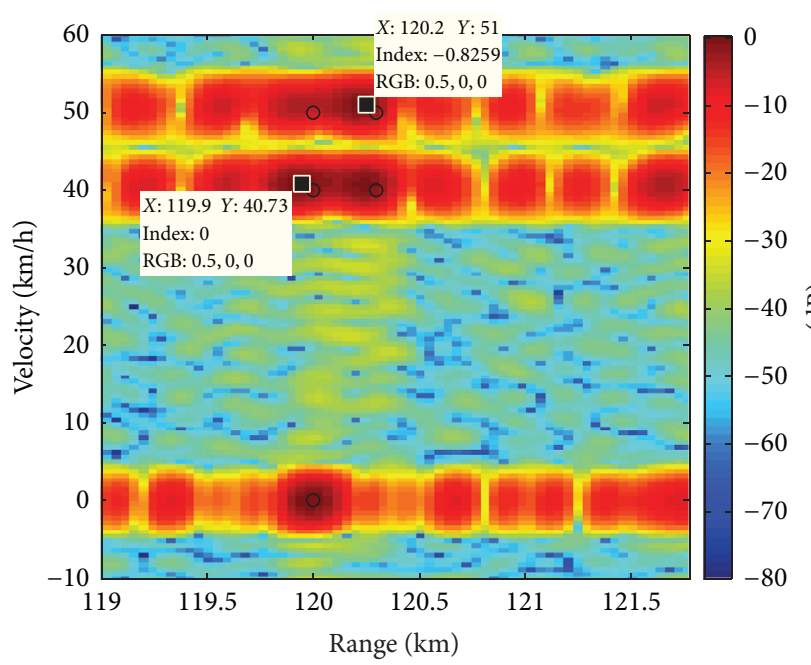

(a) Precompensation

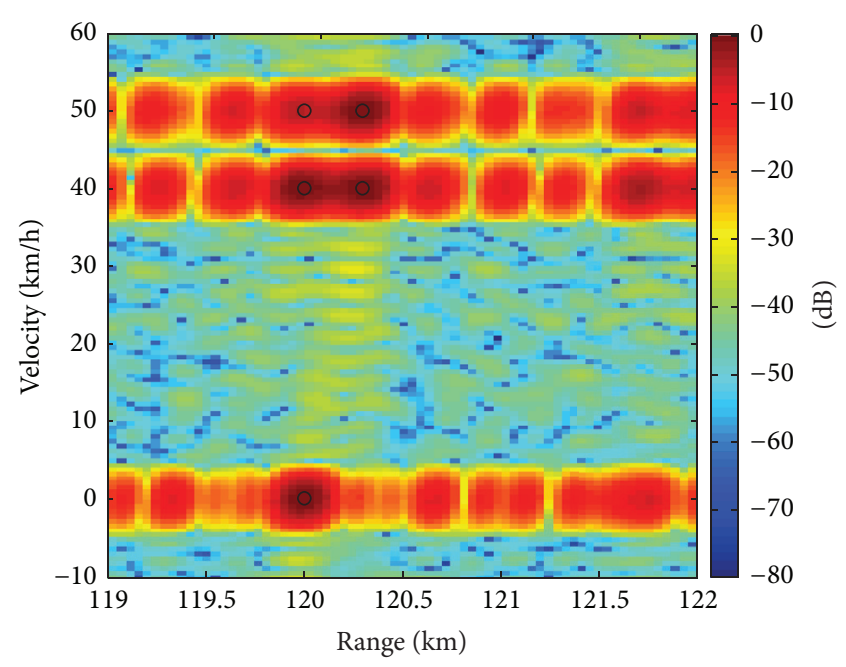

(b) Postcompensation

FIGURE 11: Range-velocity image generated by periodogram.

is a stationary one for comparison. We process 256 pulses in a single CPI, which results in an integration time of 32.8 seconds and a velocity resolution of about $3 \mathrm{~km} / \mathrm{h}$. A Taylor window with sidelobe level set to $-35 \mathrm{~dB}$ is used in the matched filtering of velocity domain.

Figure 11 is the range-velocity spectrum generated by periodogram, where the circles represent the true positions of the targets in the range-velocity space. The heavy leakage problem in the range domain is clearly illustrated in the figure.

We also show the indispensability of the intersweep and in-sweep compensation as mentioned in Section 3. Figure $11(\mathrm{a})$ is the result with neither compensation applied. The data cursors mark two of the spectrum peaks of the moving targets. The peaks of the four moving targets have several bins shifted from the truth in both range and velocity domain while peak position of the stationary one is accurate. After applying both the intersweep and in-sweep compensation, as shown in Figure 11(b), the peak positions of all the five targets turn out to be accurate.

Figure 12 is the range-velocity spectrum formed by IAA. It shows that IAA significantly outperforms periodogram both in main lobe width and in sidelobe levels which result in a higher resolution and a much better detection performance.

Similar to Figure 11, we also lay out the processing result with and without the velocity compensation. It shows that 


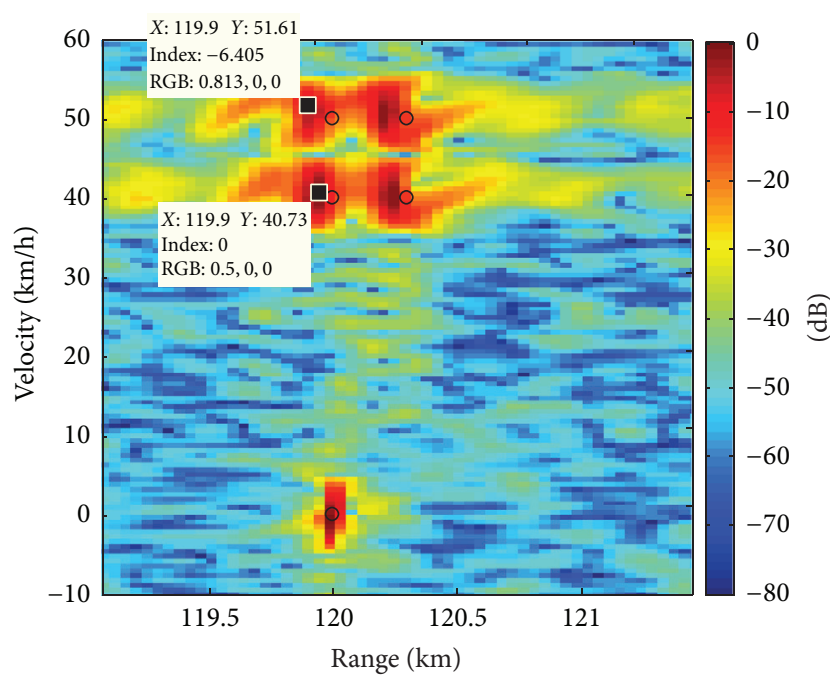

(a) Precompensation

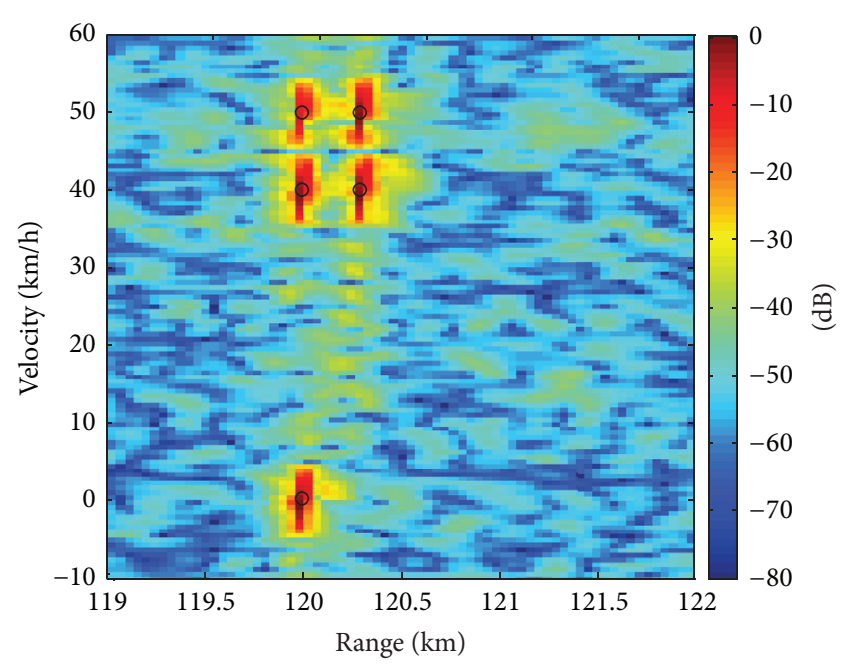

(b) Postcompensation

FIGURE 12: Range-velocity image generated by IAA.
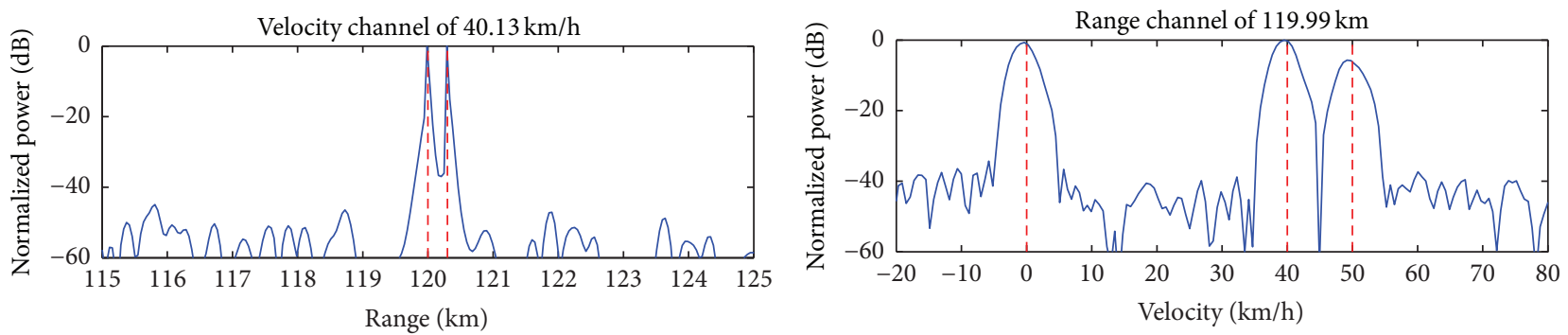

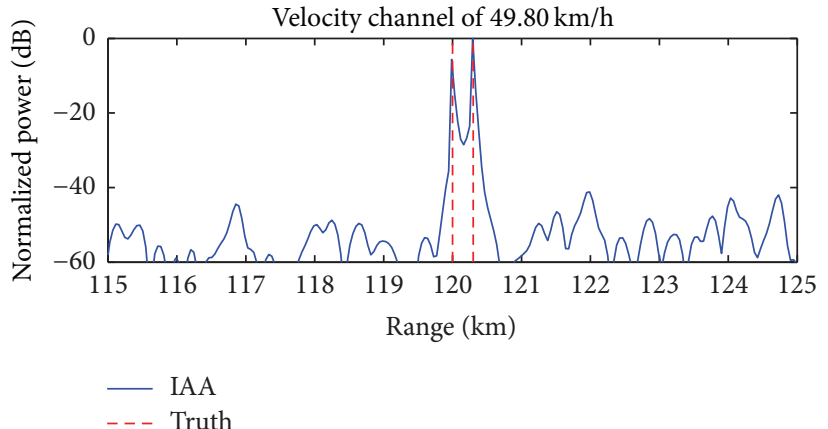

(a) Range profile

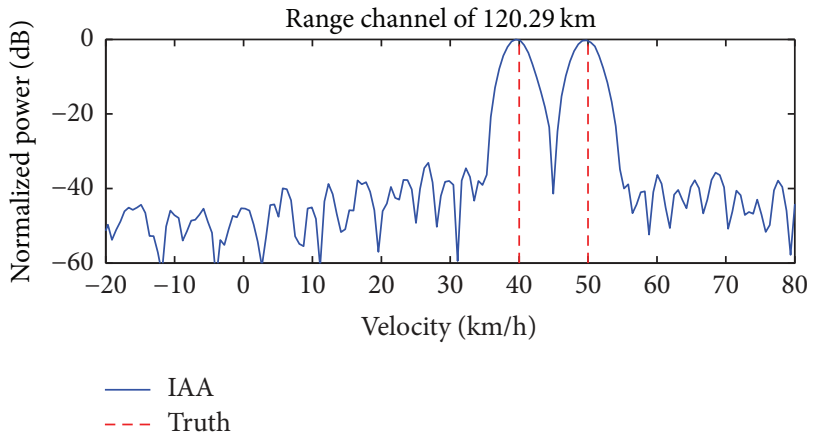

(b) Velocity profile

FIGURE 13: Range and velocity profile of postcompensation IAA spectrum at the target positions.

the IAA spectrum of moving targets suffers from a range and velocity bias and range migration without the velocity compensation. However, with the velocity compensation, the range migration phenomenon is eliminated, and the IAA spectrum has a sharp peak located precisely at the target positions. And, finally, Figure 13 shows the range and velocity profile of the postcompensation range-velocity image formed by IAA at the target positions. The closely spaced targets are properly resolved.

\section{Conclusion}

DS-FMCW signal is a variation of the conventional FMCW signal. It uses several discontinuous narrow bands to synthesize a waveform with desired bandwidth and is able to achieve the same range resolution as a full-band conventional FMCW signal in the same frequency span. This paper has presented a general range-velocity processing scheme for the DSFMCW signal. The sequence of processing steps is adjusted 
with velocity domain processing coming first, followed by range domain processing in each velocity channel. For the range domain processing, we propose a time rearrangement operation which converts the range transform problem to a general spectral estimation problem of a nonuniformly sampled data sequence. We have presented the periodogram range spectrum and the IAA range spectrum. IAA is shown to outperform the periodogram method both in resolution and in sidelobe levels. And IAA has a quite ideal spectrum output with sharp peaks at the target positions and nearzero values at other positions. The Monte Carlo simulation shows that, with a waveform OFR above $40 \%$ and a signal SNR above $0 \mathrm{~dB}$, the range spectrum of the DS-FMCW signal generated by IAA can achieve quite a low sidelobe level and an acceptable resolving ability. For the velocity processing, we propose a strategy of velocity compensation which includes the intersweep and in-sweep compensation. Finally, a complete design example showed the indispensability of the velocity compensation and validated the effectiveness of the proposed range-velocity processing scheme.

\section{Competing Interests}

The authors declare that there are no competing interests regarding the publication of this paper.

\section{References}

[1] D. E. Barrick, "History, present status, and future directions of HF surface-wave radars in the U.S.", in Proceedings of the International Radar Conference, pp. 652-655, Adelaide, Australia, September 2003.

[2] A. M. Ponsford and J. Wang, "A review of high frequency surface wave radar for detection and tracking of ships," Turkish Journal of Electrical Engineering and Computer Sciences, vol. 18, no. 3, pp. 409-428, 2010.

[3] H. J. Roarty, D. E. Barrick, J. T. Kohut, and S. M. Glenn, "Dualuse of compact HF radars for the detection of midand large-size vessels," Turkish Journal of Electrical Engineering \& Computer Sciences, vol. 18, no. 3, pp. 373-388, 2010.

[4] D. E. Barrick, J. M. Headrick, R. W. Bogle, and D. D. Crombie, "Sea backscatter at HF: interpretation and utilization of the echo," Proceedings of the IEEE, vol. 62, no. 6, pp. 673-680, 1974.

[5] K.-W. Gurgel, H.-H. Essen, and T. Schlick, "HF surface wave radar for oceanography-a review of activities in Germany," in Proceedings of the International Radar Conference (RADAR '03), pp. 700-705, Adelaide, Australia, September 2003.

[6] K.-W. Gurgel and G. Antonischki, "Measurement of surface current fields with high spatial resolution by the HF radar WERA," in Proceedings of the IEEE International Geoscience and Remote Sensing Symposium (IGARSS '97), vol. 4, pp. 1820-1822, August 1997.

[7] H. W. Leong and B. Dawe, "Channel availability for east coast high frequency surface wave radar systems," Tech. Rep., Defence R\&D, Toronto, Canada, 2001.

[8] A. Dzvonkovskaya and H. Rohling, "Software-improved range resolution for oceanographic HF FMCW radar," in Proceedings of the 14th International Radar Symposium (IRS '13), pp. 411-416, June 2013

[9] V. M. Kutuzov, "Synthesis of non-regular multitone signals and algorithms of their processing," in Proceedings of the $3 \mathrm{rd}$
International Conference on Signal Processing (ICSP '96), vol. 1, pp. 813-816, October 1996.

[10] S. D. Green and S. P. Kingsley, "Improving the range/time sidelobes of large bandwidth discontinuous spectra HF radar waveforms," in Proceedings of the 7th International Conference on HF Radio Systems and Techniques, pp. 246-250, July 1997.

[11] S. D. Green and S. P. Kingsley, "Investigation of wide bandwidth HF radar waveforms," in Proceedings of the IEE Electronics Division Colloquium on Advanced Transmission Waveforms, pp. 2/1-2/8, June 1995.

[12] Q. Wang, Z. Yang, X. Wan, and J. Xiong, "Range sidelobes suppression for HF surface wave radar with discontinuous spectra," in Proceedings of the 8th International Symposium on Antennas, Propagation and EM Theory (ISAPE '08), pp. 645648, Beijing, China, November 2008.

[13] J. Yu and J. Krolik, "Multiband chirp synthesis for frequencyhopped FMCW radar," in Proceedings of the 43rd Asilomar Conference on Signals, Systems and Computers, pp. 1315-1319, November 2009.

[14] M. J. Lindenfeld, "Sparse frequency transmit and receive waveform design," IEEE Transactions on Aerospace and Electronic Systems, vol. 40, no. 3, pp. 851-861, 2004.

[15] G. Wang and Y. Lu, "Designing single/multiple sparse frequency waveforms with sidelobe constraint," IET Radar, Sonar and Navigation, vol. 5, no. 1, pp. 32-38, 2011.

[16] W. X. Liu, Y. L. Lu, and M. Lesturgie, "Optimal sparse waveform design for HFSWR system," in Proceedings of the International Conference on Waveform Diversity and Design (WDD '07), pp. 127-130, IEEE, Pisa, Italy, June 2007.

[17] Y. Wei and Y. Liu, "New anti-jamming waveform designing and processing for HF radar," in Proceedings of the CIE International Conference on Radar Proceedings, pp. 281-284, October 2001.

[18] D. Zhang and X. Liu, "Range sidelobes suppression for wideband randomly discontinuous spectra OTH-HF radar signal," in Proceedings of the IEEE Radar Conference, pp. 577-581, April 2004.

[19] D. E. Barrick, "FM/CW radar signals and digital processing," Tech. Rep., National Oceanic and Atmospheric Administration, Boulder, Colo, USA, 1973.

[20] H. C. Chan, "Evaluation of the FMICW waveform in HF surface radar applications," Tech. Rep., Defence Research Establishment, Ottawa, Canada, 1994.

[21] P. Babu and P. Stoica, "Spectral analysis of nonuniformly sampled data-a review," Digital Signal Processing, vol. 20, no. 2, pp. 359-378, 2010.

[22] T. Yardibi, J. Li, P. Stoica, M. Xue, and A. B. Baggeroer, "Source localization and sensing: a nonparametric iterative adaptive approach based on weighted least squares," IEEE Transactions on Aerospace and Electronic Systems, vol. 46, no. 1, pp. 425-443, 2010.

[23] P. Stoica, J. Li, and H. He, "Spectral analysis of nonuniformly sampled data: a new approach versus the periodogram," IEEE Transactions on Signal Processing, vol. 57, no. 3, pp. 843-858, 2009.

[24] M. A. Richards, Fundamentals of Radar Signal Processing, McGraw-Hill, New York, NY, USA, 2nd edition, 2014.

[25] L. Eyer and P. Bartholdi, "Variable stars: which Nyquist frequency?" Astronomy and Astrophysics Supplement Series, vol. 135, no. 1, pp. 1-3, 1999. 


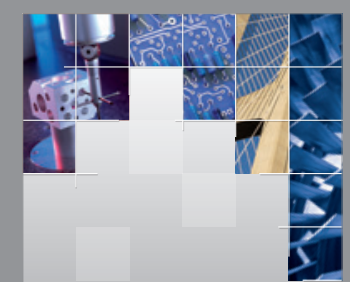

\section{Enfincering}
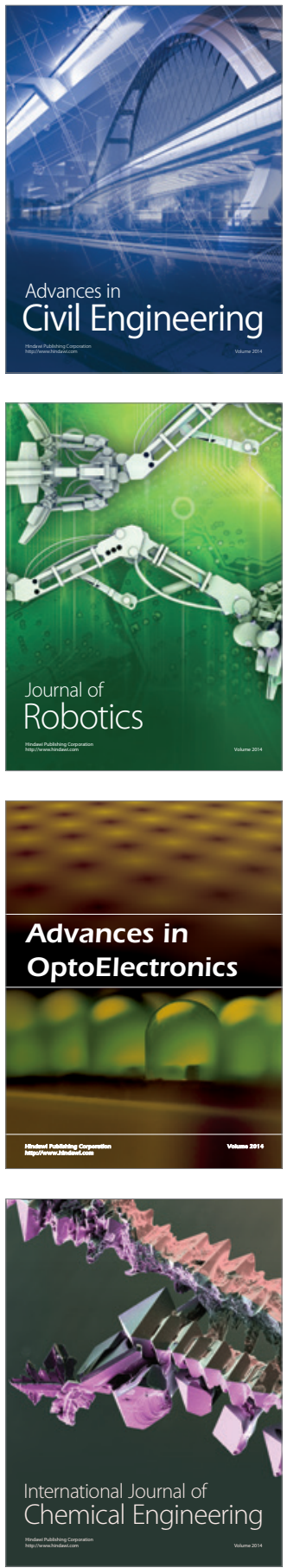

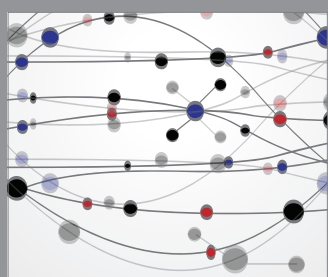

The Scientific World Journal

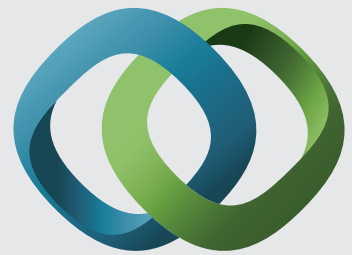

\section{Hindawi}

Submit your manuscripts at

http://www.hindawi.com
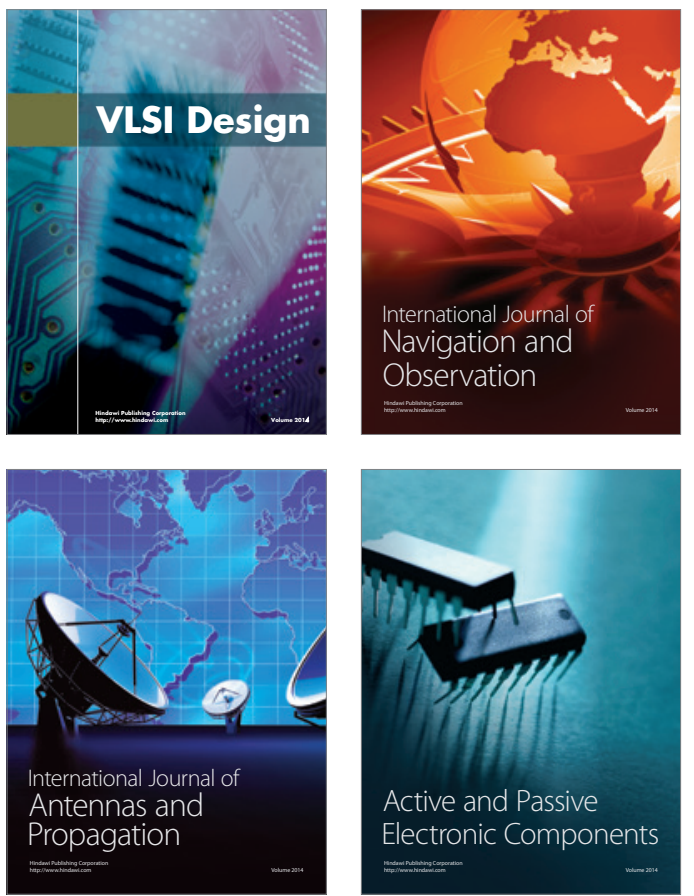
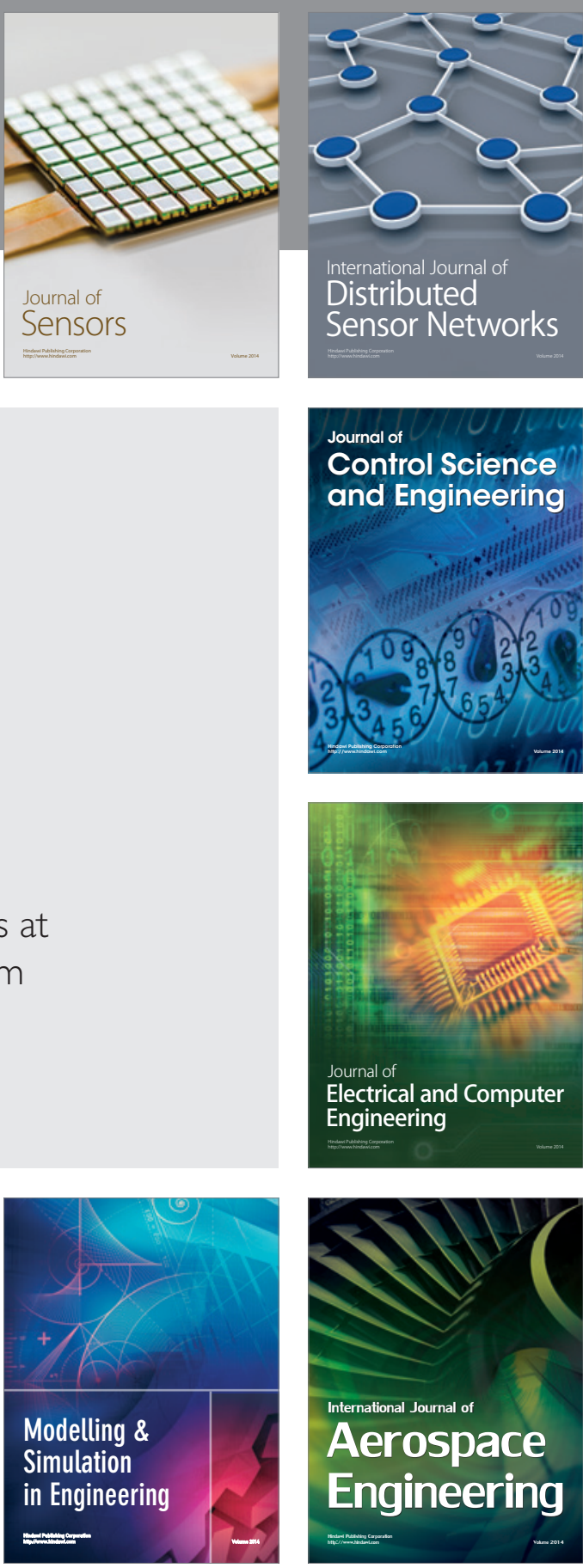

International Journal of

Distributed

Sensor Networks

Journal of

Control Science

and Engineering
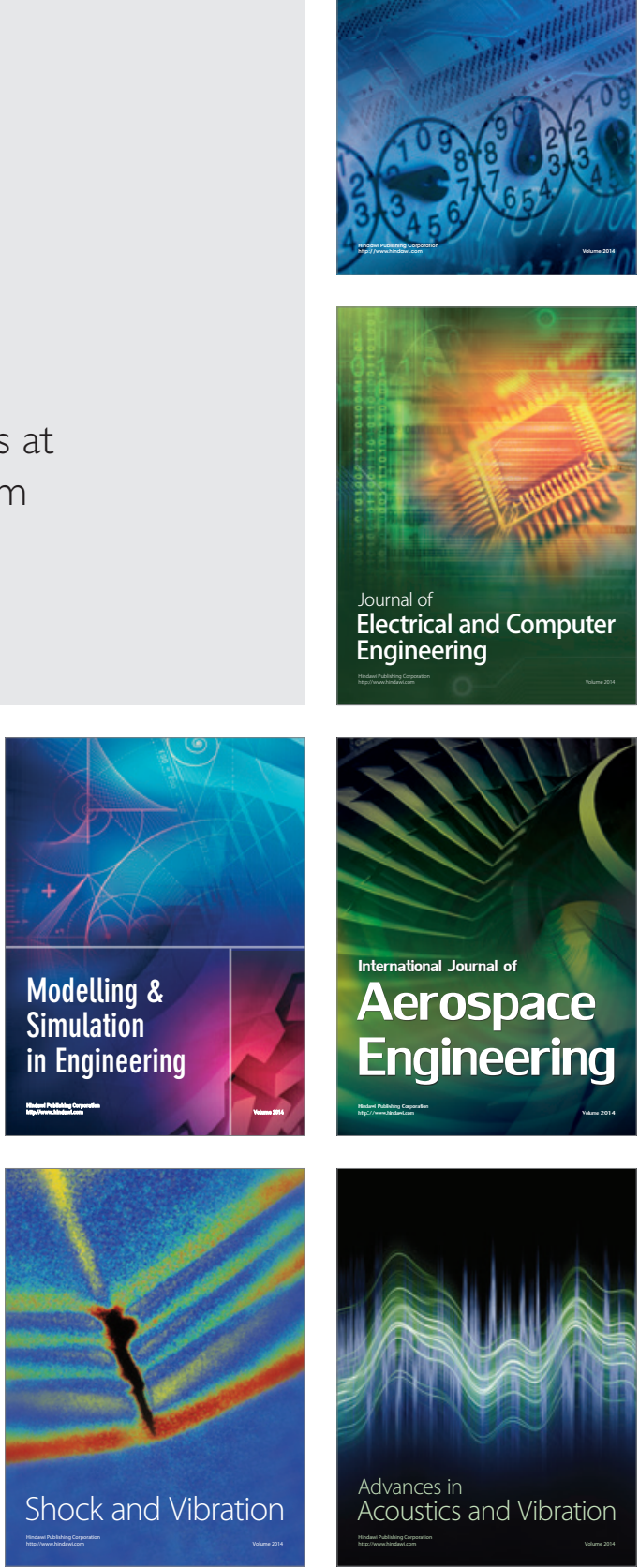